\title{
Two-stage Glass Molding of Aspheric Lens
}

\author{
K.J. Zhu ${ }^{1,2, *}$, S. Shi ${ }^{1,2}$, Z.G. Chen ${ }^{1,2}$, B. Luo ${ }^{1,2}$ and Y.Y. Zhang ${ }^{3}$ \\ ${ }^{1}$ College of Mechanical and Energy Engineering, Shaoyang University, Shaoyang, Hunan, 422000, China \\ ${ }^{2}$ Key Laboratory of Hunan Province for Efficient Power System and Intelligent Manufacturing, Shaoyang University, Hunan, 422000, \\ China \\ ${ }^{3}$ College of Optical Sciences, University of Arizona, Tucson, Arizona 85719, USA
}

Received 1 February 2020, Accepted 11 April 2020

\begin{abstract}
The glass molding has recently emerged as a promising way to mass-produce aspheric lens but has low molding accuracy. This study proposed a novel two-stage glass molding technology on the basis of the best-fitting sphere theory to improve the molding accuracy of aspheric lenses. A method of profile deviation prediction for the two-stage glass molding of aspheric lens was established on the basis of the finite element theory. Moreover, the influence of the two-stage molding temperature on profile deviation was discussed. Finally, the proposed two-stage glass molding technology was compared with the traditional glass molding process. Results demonstrate that the radius of the best-fitting sphere is $3.313 \mathrm{~mm}$ by fitting through the least squares method. When the molding temperature in the first stage is higher than that of the second stage, the molding accuracy of the aspheric lens is improved. Compared with the method of one-stage glass molding, the method of two-stage glass molding decreases the maximum profile deviation of the aspheric surface from $1.32 \mu \mathrm{m}$ to $0.83 \mu \mathrm{m}$. The service life of the aspheric mold is prolonged because most of the deformations required for glass molding are completed by the spherical mold. This study can provide references to improve the molding accuracy and reduce the production costs of the glass molding of aspheric lens.
\end{abstract}

Keywords: Aspheric lens, The two-stage glass method, Simulation prediction, Molding accuracy

\section{Introduction}

The aspheric optical lens can effectively reduce distortion and aberration, improve imaging quality, enhance system identification capabilities, and has attracted considerable attention in the field of optical component processing [1-2]. The single removal processing method is generally used in the production of traditional small-aperture aspheric lens. After multiple processes, such as fine grinding, polishing, and edging, long production cycle and high production cost exist, thereby hardly meeting the growing market demand [3-4]. With the advantages of low cost, simple process, and suitability for mass production, the glass molding technology has been widely used in the field of smallaperture aspheric glass lens manufacturing [5-6].

The traditional glass molding process is one-stage glass molding. The spheric glass preform is placed in the aspheric mold cavity and directly pressed into a predesigned aspheric shape through heating, molding, annealing, and cooling. The deformation of the spheric preform is pressed using the aspheric mold [7]. The aspheric mold and the hightemperature viscoelastic glass have long contact time, the aspheric mold got large acting force because the force is interactive, thereby shortening the service life of mold and increasing the production cost [8]. In addition, the glass preform has a large nonlinear deformation during the onestage molding process, the resilience and the residual stress increase. In the process of annealing, the profile deviation

\footnotetext{
*E-mail address: _kkjzhc1098@126.com

ISSN: 1791-2377 @ 2020 School of Science, IHU. All rights reserved.

doi:10.25103/jestr.132.09
}

caused by structure relaxation is increased which reduces the molding accuracy [9].

Considering the low molding accuracy of aspheric lens, scholars have carried out studies on the novel glass molding technology and parameter optimization to improve the molding accuracy [10-14]. However, the novel molding process is complex and cannot be accurately controlled. Also, parameter optimization has limited the effects on improved molding accuracy, which hinders the popularization and the application of the aspheric lens precision glass molding technology in the industrial field. Therefore, the manufacture of high-precision aspheric lens at low cost and high efficiency is still challenging.

To this end, this study proposed a novel two-stage glass molding technology. First, the spherical mold was manufactured on the basis of the best-fitting sphere theory at high temperature to perform the first-stage glass molding and complete most deformations of the spherical preform. Second, the aspheric mold was used to complete the final deformation. This study aimed to explore the influence of two-stage glass molding process parameters on molding accuracy and compare the proposed two-stage glass molding technology with the one-stage glass molding technology. Results can provide a reference for the low-cost batch manufacturing of high-precision aspheric lens.

\section{State of the Art}

The glass molding technology is first proposed by Eastman Kodak [15] and has developed for more than 40 years. The studies on glass molding have started earlier in foreign 
countries. Universities and research institutions in the United States, Germany, and Japan have dominated the basic mechanism study of glass molding. Toshiba, Panasonic, Canon of Japan, Kodak, Rochester, Moore of the United States, Daeho of South Korea, SCHOTT and ZEISS of Germany, Foxconn and Sunny of China have led the product-targeted action-oriented study.

Firstly, the mechanism of glass molding was studied. Shishido [16] investigated the influence of the bonding degree between the glass preform and the mold on molding accuracy and found that a high bonding degree can improve the molding accuracy of the aspheric lens. Hosoe [17] improved production efficiency and reduced costs through the independent distribution of multiple molds, mold-tomold combinations, and improved heating system, thereby enhancing the molding accuracy to some extent.

Mosaddegh [18] and Ananthasayanam [19] tested the friction behavior between the glass preform and the mold surface in glass molding and discussed the influence of interface friction behavior on molding accuracy. Klocke [20] performed SEM/EDX and X-ray photoelectron spectroscopy of precious metal PVD coating on the mold surface and studied the changes of the wear and the composition in different surface areas. Moreover, Klocke proposed a qualitative analysis model for coating failure, which could provide a reference to improve the service life of molds. Zhou [21] studied the thermoviscoelastic behavior of glass near the molding temperature, discussed the effects of creep and stress relaxation behavior on molding accuracy. The above-mentioned studies have achieved considerable results in terms of glass molding mechanism, which provide theoretical guidance for the subsequent process parameter optimization. However, few studies involve improving the molding accuracy.

Scholars also studied the parameters optimization to improve the molding accuracy. Yi [22] established a finite element numerical model on the base of experimental measurement results, discussed the influence of molding parameters, such as molding temperature, molding rate and annealing rate on residual stress and profile deviation. Sarhadi [23] used the FORTRAN user subroutine to establish a three-dimensional thermodynamic model and explored the influence of molding parameters, such as molding temperature and cooling rate on molding accuracy by using the ABAQUS. The process parameters were further optimized to improve the shape accuracy of the lens. Mahajan [24] repeatedly optimized the profile of mold surface by measuring the profile deviation of the molded lens surface and used the finite element method to perform the simulation optimization analysis. The shape accuracy of the aspheric surface reached submicron accuracy. Tao [25] optimized the annealing process to further improve the molding accuracy. Huo [19] used the single factor analysis method to study the influence of the molding process parameters on profile deviation and conducted a preliminary optimization analysis. Yin [26] discussed the finite element simulation modeling theory of glass molding, established the finite element models for heating stage, molding stage, and annealing stage, systematically analyzed the influence of molding parameters, such as molding temperature, molding rate and annealing rate on profile deviation. Su [27] explored the influence of the thermal expansion coefficient, cooling rate, and holding force on the profile deviation of lens. On the basis of the numerical calculations of thermal shrinkage, volume change, and stress distribution, Liu [28] predicted the profile deviation of the molded glass optical elements before carrying out the molding experiment. The simulation results were consistent with the molding experiment results. Liu [29] thoroughly analyzed the optimization of the process parameters of chalcogenide glass. The above-mentioned scholars have improved the molding accuracy to some extent but only reduced the deviation value, and the improvement degree is still limited.

Considering the above-mentioned reasons, scholars have carried out exploratory studies focusing on the new technology of glass molding. Zhou [30] and Yu [31] used the ultrasonic-assisted glass molding method to improve the molding accuracy, deeply analyzed the mechanism of reducing the interface friction resistance and ultrasonicassisted filling. The effectiveness of improving the molding accuracy was verified. However, the ultrasonic-assisted mechanism is still unclear, it is difficult to form an integrated system effectively, and the production cost is high. $\mathrm{Vu}$ [32] studied the vacuum-assisted glass molding technology, which was compared with traditional processes to verify the effectiveness of improving molding accuracy. However, the cost of vacuum environment is high, and realizing a high-temperature sealing is difficult.

The above-mentioned studies focused on the optimization of molding process parameters, but the improvement degree of the molding accuracy is relatively limited. Moreover, exploratory studies were carried out on the new glass molding technology, which has some problems, such as high cost, and it is difficult to be widely used in enterprises. This study proposed a novel two-stage glass molding method on the basis of the best-fitting sphere theory. First, the best-fitting sphere mold is used to perform the first-stage glass molding which complete most of the deformations of the spherical preform. Second, an aspheric mold was used to achieve the final deformation, thereby improving the molding accuracy and prolonging the service life of the difficult-to-machine aspheric molds. The proposed method can provide a reference for improving molding accuracy.

The remainder of the study is organized as follows. The best-fitting spherical solution method for a given aspheric design curve and the simulation analysis theory are described in Section 3. In Section 4, the finite element method is used to establish a profile deviation prediction model for two-stage glass molding, the effects of molding temperature on profile deviation at the first stage and the second stage are analyzed. Moreover, the proposed molding method is compared with the traditional one-stage glass molding. The summary and conclusions of this study are presented in the last section.

\section{Methodology}

\subsection{Design of Double Aspheric Lens}

A single-sided aspheric lens was selected. The upper surface is a flat surface, and the lower surface is an aspheric surface. The cross-sectional curve is defined as follows (Equation [1]):

$$
\begin{aligned}
& z=f(x)=\frac{x^{2} / R}{1+\sqrt{1-(k+1)(x / R)^{2}}}+\sum_{i=2}^{n} B_{2 i} \cdot x^{2 i} \\
& \sum_{i=2}^{n} B_{2 i} \cdot x^{2 i}=A_{4} x^{4}+A_{6} x^{6}+A_{8} x^{8}+A_{10} x^{10}
\end{aligned}
$$


where $x$ is the coordinate value of curve $\mathrm{X}, z$ is the vector function of $\mathrm{X}, r$ is the curvature radius of the vertex of the curve, $\sum_{i=2}^{n} B_{2 i} \cdot x^{2 i}$ is the aspheric correction term, $k$ is the quadratic coefficient. The parameters of the convex surface are as follows: $R=2.2 \mathrm{~mm}, k=-2.64, \mathrm{~A}_{4}=2.468 \times 10^{-3}$, $\mathrm{A}_{6}=-2.852 \times 10^{-3}, \mathrm{~A}_{8}=4.229 \times 10^{-4}$, and $\mathrm{A}_{10}=4.749 \times 10^{-5}$.

\subsection{Two-stage glass molding and best-fitting spherical solution}

The two-stage glass molding process of the aspheric glass lens is shown in Fig.1. In the first glass molding stage, the spheric glass preform was first pressed to the best-fitting spheric surface. The best-fitting spheric lens was then pressed to the designed aspheric lens through the second glass molding. The traditional one-stage glass molding process was decomposed into two stages. The spheric glass was first pressed into the best-fitting spheric surface and then pressed into the final aspheric shape.

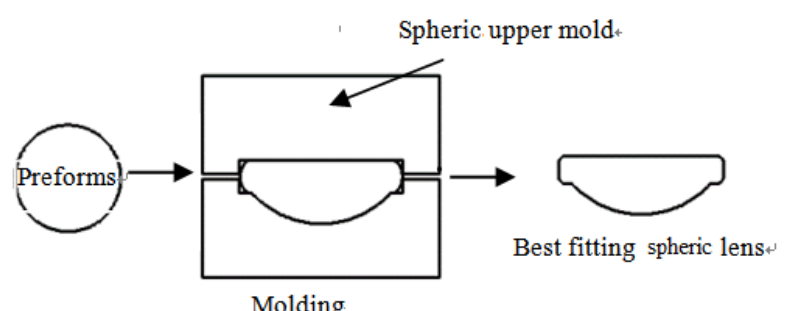

(a)

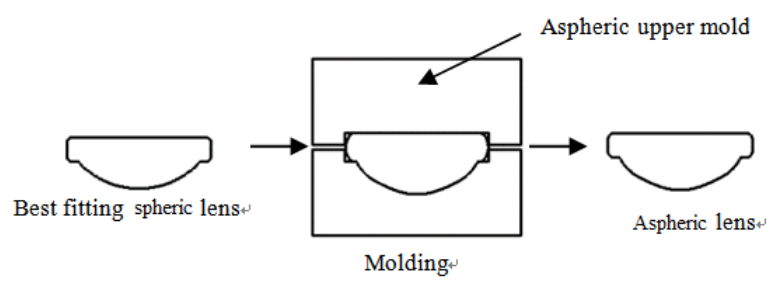

(b)

Fig.1. Two-stage glass molding of the aspheric glass lens. (a) First-stage glass molding. (b) Second-stage glass molding

On the base of the aspheric cross-section curve equation, the circle-fitting function was used to fit in the MATLAB. The radius of the best-fitting sphere is $3.313 \mathrm{~mm}$. The bestfitting spheric and the aspheric curves were shown in Fig.2.

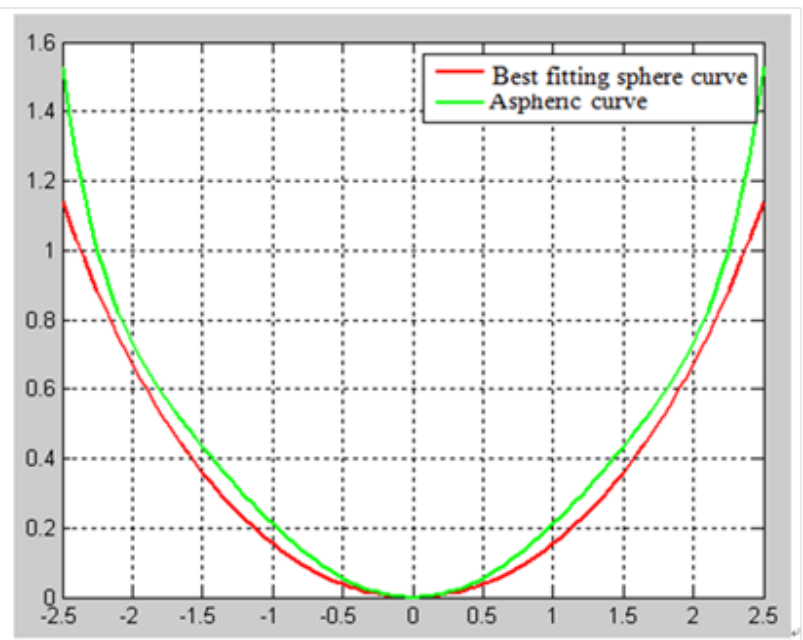

Fig. 2. Aspheric and best-fitting spherical curves

\subsection{Establishment of the two-stage glass molding} simulation model

The designed best-fitting spherical curve was input into $\mathrm{CAD}$, and the geometric model closest to the spheric mold was drawn. The model was introduced into the Marc operation interface to establish the 2D axisymmetric numerical simulation model in the first glass molding stage (Fig. 3).

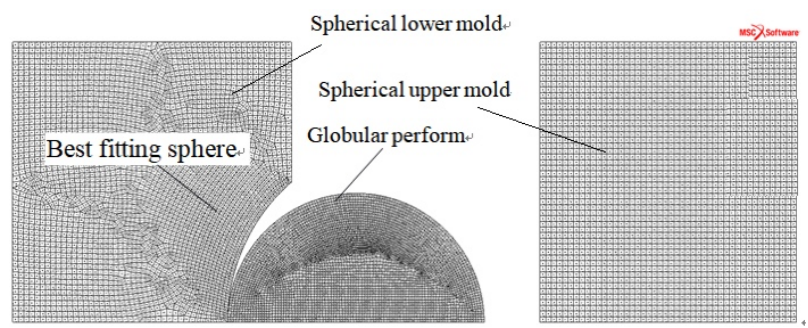

Fig. 3. Finite element model of the first glass molding

The first glass molding is the basis of the second glass molding process. The prediction results of the best-fitting spheric lens are extracted and imported into the second glass molding model (Fig.4).
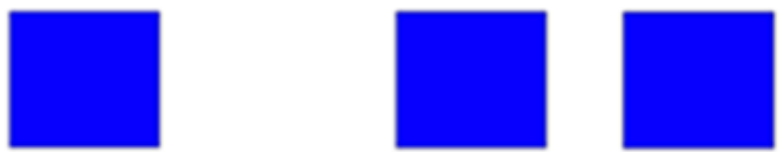

Extractioniing

Importing
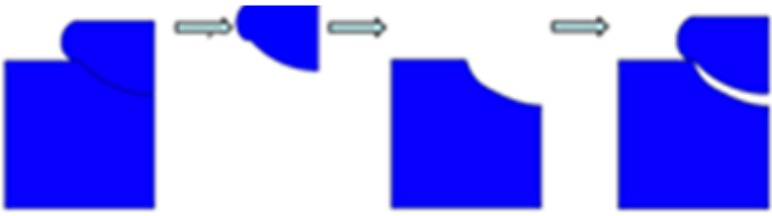

Fig. 4. Extraction and import of the best-fitting spheric lens

\section{Result Analysis and Discussion}

\subsection{Simulation analysis of the two-stage glass molding} In the first glass molding simulation process, a thermal boundary condition was added to the side of the mold, thereby providing a continuous heating source. A thermal boundary condition was added to the bottom surface of the mold in the $\mathrm{X}$ direction, and a fixed boundary condition was added to the side of the mold in the $\mathrm{Y}$ direction. The established simulation analysis model is shown in Fig.5.

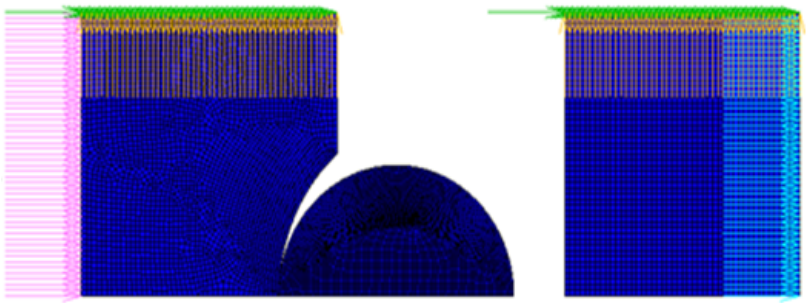

Fig. 5. Boundary conditions in the first glass molding heating stage

Fig. 6 shows the best-fitting spherical lens molding process at the first glass molding. After the heating stage of glass molding, the internal temperature of the preforms is the same. Fig.6 (a) shows that the lower mold moved quickly upward under the boundary conditions of the molding pressure. After the glass preform came in contact with the 
upper mold, the glass was pressed into the best-fitting spherical glass lens at a prescribed molding rate. Fig.6 (b) shows that the lens was processed to the designed shape, and the first glass molding process was finished.

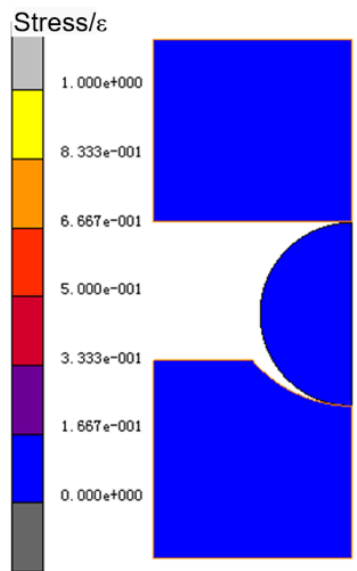

(a)

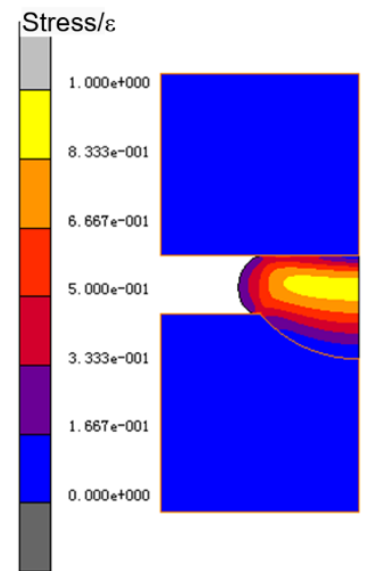

(b)
Fig .6. Simulation analysis of the molding stage of the first glass molding. (a) Upper surface coming in contact with the mold. (b) glass molding

During the annealing stage, the molded glass lens gradually cooled, and the internal structural relaxation occurred, which evidently influenced the overall lens shape and the spheric molding accuracy. The annealing stage was analyzed by taking the postprocessing file generated at the molding stage as the model at the annealing stage, which was reimported to Marc. The boundary conditions were applied to the model, as shown in Fig.7.
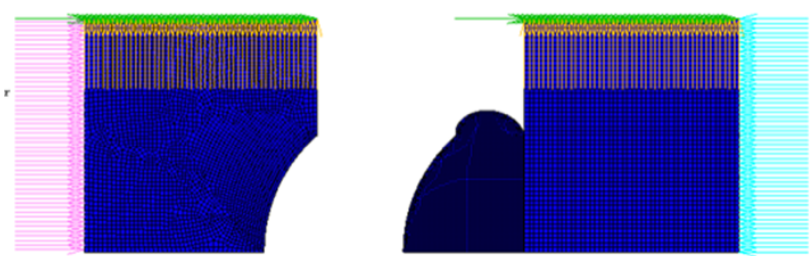

Fig. 7. Boundary conditions at the annealing stage of the first glass molding

The prediction results of the best-fitting spherical lens in the first glass molding stage were extracted and introduced into the second glass molding model and the thermal and force boundary conditions were set. The simulation model is shown in Fig.8.
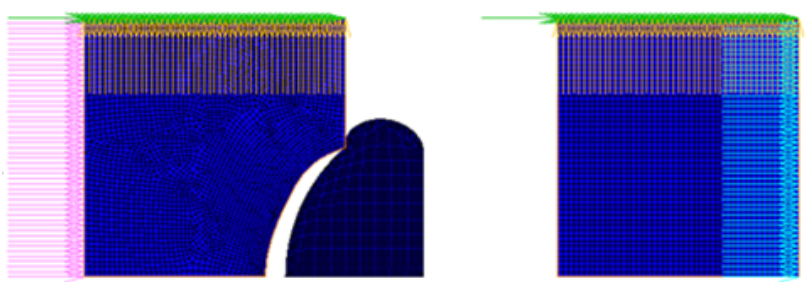

Fig. 8. Simulation model of the second glass molding stage

Fig.9 shows the aspheric lens glass molding process during the molding stage at the second glass molding stage, which is similar to that at the first glass molding stage. Fig.9 (a) shows the initial stage of the second glass molding process, and the temperature in the best-fitting spherical lens is the same. Fig. 9 (b) shows that the lower mold moved quickly upward under the effect of pressure boundary conditions. After the best-fitting spherical lens came in contact with the upper mold, the glass preform was pressed to the designed aspheric lens at the prescribed molding rate. As shown in Fig. 9 (c), the deformation in the transformation from the best-fitting spherical lens to the aspheric lens was small, and the residual stress was significantly reduced, thereby improving the surface accuracy of the aspheric lens. Fig.9 (d) shows that the lens was processed to the predetermined shape, and the glass molding phase ended.

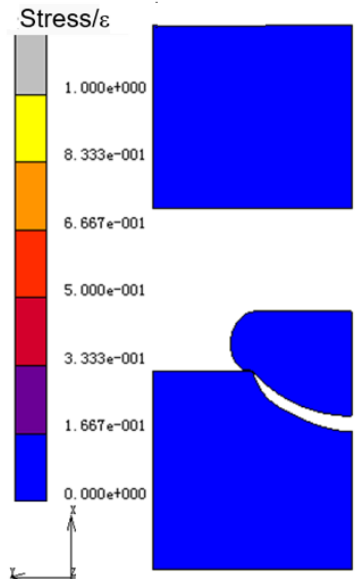

(a)

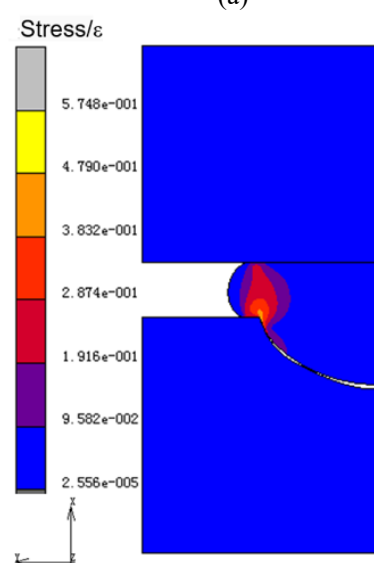

(c)

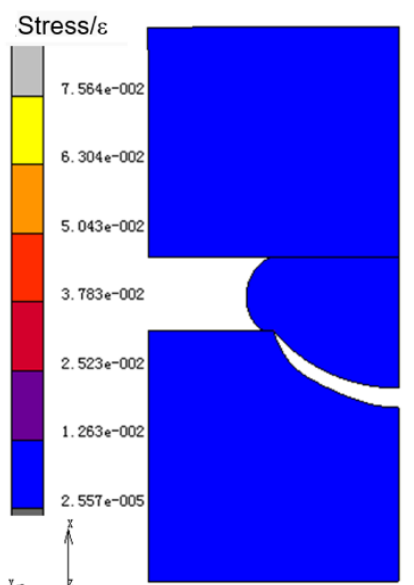

(b)

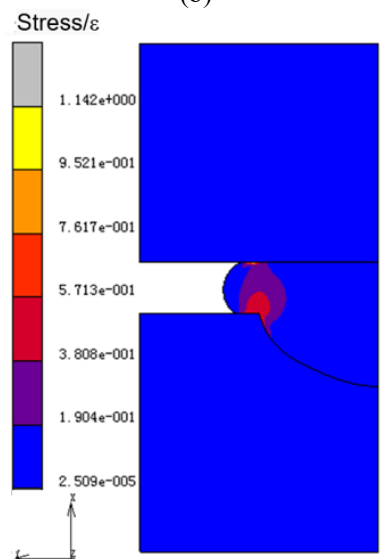

(d)
Fig. 9. Simulation analysis of the molding stage of the second glass molding. (a) Start of glass molding. (b) Upper surface coming in contact with the mold. (c) Glass molding. (d)Completion of glass molding

\subsection{Analysis of the two-stage molding temperature}

In the two-stage glass molding process, the setting of the molding temperature was important. Given that the first glass molding stage aimed to press the spherical preform into the best-fitting spherical shape, the deformation to be completed was large, and a high molding temperature was suitable. The deformation in the second glass molding stage was small, and low molding temperature should be set to improve the molding accuracy. On the basis of the above design purposes, the molding temperatures at the first and the second stage was set to $580{ }^{\circ} \mathrm{C}-560{ }^{\circ} \mathrm{C}, 570{ }^{\circ} \mathrm{C}-570{ }^{\circ} \mathrm{C}$, and $560{ }^{\circ} \mathrm{C}-580{ }^{\circ} \mathrm{C}$. The simulation analysis results were processed to obtain the profile deviation of the aspheric lens (Fig.10).

As shown in Fig.10, at the molding temperature of $560{ }^{\circ} \mathrm{C}-580{ }^{\circ} \mathrm{C}$, the overall profile deviation of the obtained aspheric lens was the largest, and the maximum profile deviation value was $1.35 \mu \mathrm{m}$. At the molding temperature of $580{ }^{\circ} \mathrm{C}-560{ }^{\circ} \mathrm{C}$, the overall profile deviation was the smallest, and the maximum profile deviation value was $0.81 \mu \mathrm{m}$. The deformation to be completed in the first glass molding stage 
was large, and a high molding temperature can effectively reduce the viscosity of glass, making the glass easily flow and deform under the action of pressure and enhancing the plasticity. At the second glass molding stage, the deformation of the best-fitting spherical glass was small, which can be achieved at low molding temperature, and the residual stress decreased, resulting in structural relaxation at the annealing stage caused by reduced profile deviation.

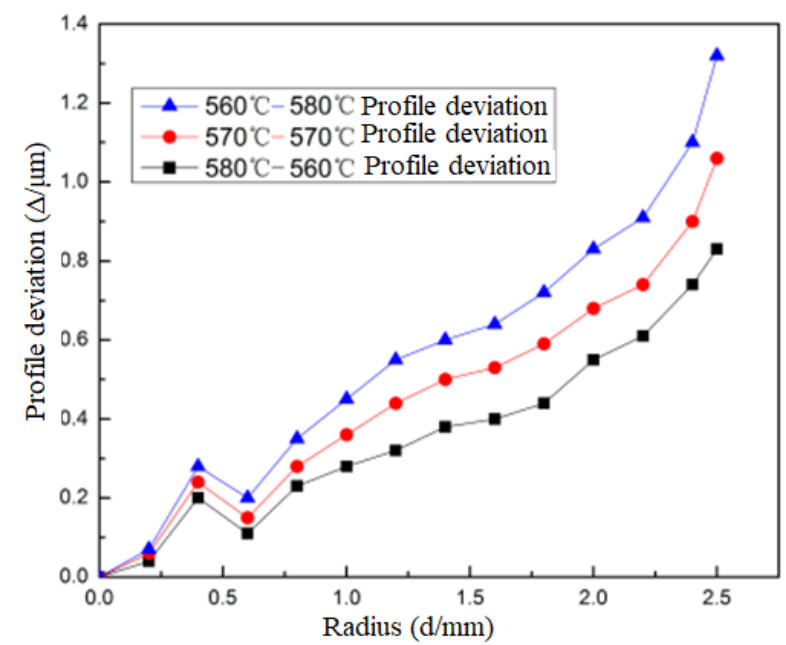

Fig. 10. Influence of hot-pressing temperature on profile deviation

\subsection{Comparative analysis of the two- and the one-stage glass molding}

The molding temperatures of the one- stage and the twostage glass molding were $580{ }^{\circ} \mathrm{C}$ and $580{ }^{\circ} \mathrm{C}-560{ }^{\circ} \mathrm{C}$, respectively, and the other parameters were as follows: molding rate was $0.02 \mathrm{~mm} / \mathrm{s}$, friction coefficient was 0.5 , annealing rate was $2^{\circ} \mathrm{C} / \mathrm{s}$, and holding pressure was $700 \mathrm{~N}$. The one- stage and the two-stage glass molding processes of aspheric lens were conducted using numerical simulation. The profile deviation curves of the obtained molded lens are shown in Fig. 11.

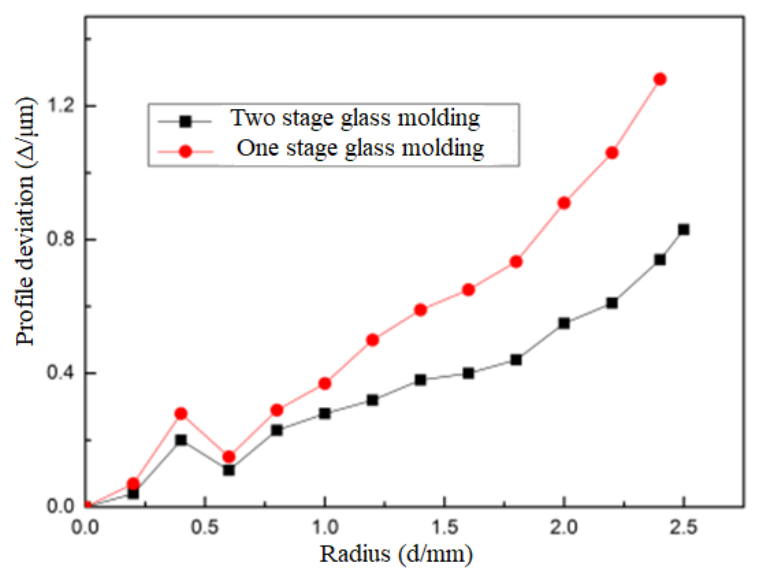

Fig. 11. Profile deviation curves of the lens obtained by one- and twostage glass molding processes

The maximum profile deviation of the aspheric lens simulated using the traditional one-stage glass molding method was $1.31 \mu \mathrm{m}$, whereas that using the two-stage glass molding method was $0.72 \mu \mathrm{m}$. Moreover, the profile deviation of the lens increased with increased centrifugal distance, showing an increasing trend. In other words, the profile deviation of the lens gradually increased from the lens center to the lens edge. Also, the lens obtained using the two-stage glass molding has lower profile deviation than that obtained using the one-stage glass molding. According to the simulation analysis results, the two-stage glass molding can effectively reduce the profile deviation and improve the molding accuracy of the aspheric lens.

\section{Conclusions}

The D-ZK3 low-melting point optical glass was used to explore the influence of the two-stage glass molding parameters on profile deviation. Based on the best-fitting spherical theory, the Marc software was used to carry out the numerical simulation analysis of the two-stage glass molding process of the D-ZK3 glass. The profile deviations of the lens obtained using the one- stage and the two-stage glass molding processes were compared. Finally, the following conclusions were drawn.

(1) The best-fitting spherical surface is determined on the basis of the originally designed aspheric surface, which is fitted in the MATLAB by using the least square method. The radius of the best-fitting spherical surface is $3.313 \mathrm{~mm}$.

(2) The molding temperature at the first glass molding stage should be higher than that at the second glass molding stage, i.e., $580{ }^{\circ} \mathrm{C}$ and $560{ }^{\circ} \mathrm{C}$, respectively, to obtain a highprecision aspheric lens.

(3) The two-stage glass molding method can effectively reduce the profile deviation and improve the molding accuracy.

(4) Because most of the deformations required for glass molding are completed using an easy-to-machine spherical mold, the service life of difficult-to-machine aspheric mold can be extended to a certain extent.

In this study, the best-fitting spherical theory was applied to the two-stage glass molding technology of aspheric glass lens. The finite element method was used to study the two stage of glass molding, and the molding temperature settings at the two stage were compared and analyzed. Results showed that the two-stage glass molding method can effectively improve the molding accuracy. This study can provide a reference for the production of high-precision aspheric lens. Given that the mold processing accuracy is limited and that the profile deviation distribution cannot be accurately controlled, it is difficult to verifying the accuracy through experiments, which should be further explored in future studies.

\section{Acknowledgements}

This work was supported by the National Natural Science Foundation of China (Grant No. 91860133) and Hunan Education Department Project (Grant No. 19B516).

This is an Open Access article distributed under the terms of the Creative Commons Attribution License 


\section{References}

1. Yan, G. P., You, K. Y., Fang, F. Z., "Three-linear-axis grinding of small aperture aspheric surfaces". International Journal of Precision Engineering and Manufacturing-Green Technology, 2019, pp.1-12.

2. Chang, K. M., Cheng, W. T., Liu, Y. T., "Development of nonaxisymmetric aspheric ultraprecision machining using FPGA-based piezoelectric FTS". Sensors and Actuators A: Physical, (291), 2019, pp.99-106.

3. Huang, C. T., Chen, L. J., Chien, T. Y., "Investigation of the Viscoelastic Behavior Variation of Glass Mat Thermoplastics (GMT) in Compression Molding". Polymers, 11(2), 2019, pp.335.

4. Jayavardhan, M. L., Kumar, B. R., Doddamani, M., "Development of glass microballoon/HDPE syntactic foams by compression molding". Composites Part B: Engineering, (130), 2017, pp.119131.

5. Zhang, L., Zhou, W., Naples, N. J., "Investigation of index change in compression molding of As40 $\mathrm{Se}_{50} \mathrm{~S}_{10}$ chalcogenide glass". Applied optics, 57(15) ,2018, pp.4245-4252.

6. Zhou, J., Yu, J., Lee, L. J., "Stress relaxation and refractive index change of As2S3 in compression molding". International Journal of Applied Glass Science, 8(3) ,2017, pp.255-265.

7. Yang, H., Wang, Z., Zhang, Y., "Precision glass molding technology for low Tg glasses". In: Society of Photo-Optical Instrumentation Engineers (SPIE) Conference Series, Xi'an, China: SPIE, 2017, pp.102563N1-7.

8. Zhang, Y., Yan, G., Li, Z., "Quality improvement of collimating lens produced by precision glass molding according to performance evaluation". Optics Express, 27(4), 2019, pp.5033-5047.

9. Zhang, Y., Yin, S., Liang, R., "New testing and calculation method for determination viscoelasticity of optical glass". Optics Express, 28(1), 2020, pp.626-640.

10. Moore, S., Gomez, J., Lek, D., "Experimental study of polymer microlens fabrication using partial-filling hot embossing technique". Microelectronic Engineering, (162), 2016, pp.57-62.

11. Friedrichs, M., Peng, Z., Grunwald, T., "PtIr protective coating system for precision glass molding tools: Design, evaluation and mechanism of degradation". Surface and Coatings Technology, (385), 2020, pp.1253781-9.

12. Pallicity, T. D., Vu, A. T., Ramesh, K., "Birefringence measurement for validation of simulation of precision glass molding process". Journal of the American Ceramic Society, 100(10), 2017, pp.46804698.

13. Zhou, T., Zhu, Z., Liu, X., “A review of the precision glass molding of chalcogenide glass for infrared optics". Micromachines, 9(7), 2018 , pp.337.

14. Yan, J., Zhou, T., Masuda, J., "Modeling high-temperature glass molding process by coupling heat transfer and viscous deformation analysis". Precision Engineering, 33(2), 2009, pp.150-159.

15. Wittwer, V., Gombert, A., Rose, K., "Applications of periodically structured surfaces on glass". Glass science and technology, 73(4), 2000, pp.116-118.

16. Shishido, K., Sugiura, M., Shoji, T., "Aspect of glass softening by master mold". In: Proceedings of the SPIE - The International Society for Optical Engineering, San Diego, USA: SPIE, 1995, pp.421-433.
17. Hosoe, S., Masaki, Y., "High-speed glass-molding method to mass produce precise optics". In: International Conference on Optical Fabrication and Testing, Tokyo, Japan: SPIE, 1995, pp.115-120.

18. Mosaddegh, P., Ziegert, J., "The effect of temperature on the stickslip friction behavior of optical glasses in precision glass molding". Applied Mechanics \& Materials, (307), 2013, pp.381-386.

19. Ananthasayanam, B., Joshi, D., Stairiker, M., "High temperature friction characterization for viscoelastic glass contacting a mold". Journal of non-crystalline solids, (385), 2014, pp.100-110.

20. Klocke, F., Dambon, O., Rohwerder, M., "Model of coating wear degradation in precision glass molding". The International Journal of Advanced Manufacturing Technology, 87(1-4), 2016, pp.43-49.

21. Zhou, T., Zhou, Q., Xie, J., "Elastic-viscoplasticity modeling of the thermo-mechanical behavior of chalcogenide glass for aspheric lens molding”. International Journal of Applied Glass Science, 9(2), 2018, pp.252-262.

22. Yi, A. Y., Jain, A., "Compression molding of aspherical glass lenses-a combined experimental and numerical analysis". Journal of the American Ceramic Society, 88(3), 2005, pp.579-586.

23. Sarhadi, A., Hattel, J. H., Hansen, H. N., "Three-dimensional modeling of glass lens molding". International Journal of Applied Glass Science, 6(2), 2015, pp.182-195.

24. Mahajan, P., Dora, P. T., Sandeep, T. S., "Optimized design of optical surface of the mold in precision glass molding using the deviation approach". International Journal for Computational Methods in Engineering Science and Mechanics, 16(1), 2015, pp.53-64.

25. Tao, B., He, P., Shen, L., "Annealing of compression molded aspherical glass lenses". Journal of manufacturing Science and Engineering, 136(1), 2014, pp.011008.

26. Yin, S., Jia, H., Zhang, G., "Review of small aspheric glass lens molding technologies". Frontiers of Mechanical Engineering, 12(1), 2017, pp.66-76.

27. Su, L. J., Wang, F., He, P., “An integrated solution for mold shape modification in precision glass molding to compensate refractive index change and geometric deviation". Optics and Lasers in Engineering, 53(1), 2014, pp.98-103.

28. Liu, Y., Xing, Y., Yang, C., "Simulation of heat transfer in the progress of precision glass molding with a finite element method for chalcogenide glass". Applied optics, 58(27), 2019, pp.73117318 .

29. Liu, W. G., Shen, P., "Viscoelastic properties of chalcogenide glasses and simulation of molding process". Infrared and Laser Engineering, (03), 2012, pp.569-574.

30. Zhou, T., Xie, J., Yan, J., "Improvement of glass formability in ultrasonic vibration assisted molding process". International Journal of Precision Engineering and Manufacturing, 18(1), 2017, pp.57-62.

31. Yu, J. W., Li, C., Xie, W. Q., "Numerical simulation on glass molding press of micro grooves". Ordnance Material Science and Engineering, (01), 2017, pp.20-24.

32. Vu, A. T., Vogel, P. A., Dambon, O., "Vacuum-assisted precision molding of 3D thin microstructure glass optics". In: Society of Photo-Optical Instrumentation Engineers Conference Series, Strasbourg, France: SPIE, 2018, pp.106830C. 